\title{
Pleural Biphasic Synovial Sarcoma
}

National Cancer Institute

\section{Source}

National Cancer Institute. Pleural Biphasic Synovial Sarcoma. NCI Thesaurus. Code C45698.

A biphasic synovial sarcoma arising in the pleural cavity. 